\title{
Analyzing Political Bias and Unfairness in News Articles at Different Levels of Granularity
}

\author{
Wei-Fan Chen \\ Paderborn University \\ Department of Computer Science \\ cwf@mail.upb. de \\ Henning Wachsmuth \\ Paderborn University \\ Department of Computer Science \\ henningw@upb. de
}

\author{
Khalid Al-Khatib \\ Bauhaus-Universität Weimar \\ Faculty of Media, Webis Group \\ khalid.alkhatib@uni-weimar.de
}

\author{
Benno Stein \\ Bauhaus-Universität Weimar \\ Faculty of Media, Webis Group \\ benno.stein@uni-weimar.de
}

\begin{abstract}
Media organizations bear great reponsibility because of their considerable influence on shaping beliefs and positions of our society. Any form of media can contain overly biased content, e.g., by reporting on political events in a selective or incomplete manner. A relevant question hence is whether and how such form of imbalanced news coverage can be exposed. The research presented in this paper addresses not only the automatic detection of bias but goes one step further in that it explores how political bias and unfairness are manifested linguistically. In this regard we utilize a new corpus of 6964 news articles with labels derived from adfontesmedia.com and develop a neural model for bias assessment. By analyzing this model on article excerpts, we find insightful bias patterns at different levels of text granularity, from single words to the whole article discourse.
\end{abstract}

\section{Introduction}

Reporting news in a politically unbiased and fair manner is a key component of journalism ethics and standards. "Politically unbiased" means to report on an event without taking a political position, characterization, or terminology, and "fair", in this context, means to focus on original facts rather than on analyses or opinion statements based on false premises. ${ }^{1}$ Although it is known that biased and unfair news do exist in the media, spreading misleading information and propaganda (Groseclose and Milyo, 2005), people are not always aware of reading biased content.

When fighting bias and unfairness, only the understanding of how these emerge (and how to avoid

\footnotetext{
${ }^{1}$ See http://www. adfontesmedia.com for a detailed characterization of political bias and unfairness.
}

them) can allow media organizations to maintain credibility and can enable readers to choose what to consume and what not. In pursuit of this goal, we recently studied how sentence-level bias in an article affects the political bias of the whole article (Chen et al., 2020). Also other researchers have proposed approaches to automatic bias detection (see Section 2 for details). However, the existing approaches lack an analysis of what makes up bias, and how it exposes in different granularity levels, from single words, to sentences and paragraphs, to the entire discourse.

To close this gap, we analyze political bias and unfairness in this paper within three steps:

1. We develop an automatic approach to detect bias and unfairness in news articles.

2. We study the bias distribution along different text granularity levels.

3. We explore various sequential patterns of how bias and unfairness become manifest in text.

We utilize two well-known websites that address media bias, allsides.com and adfontesmedia.com, in order to create a corpus with 6964 news articles, each of which is labeled for its topic, political bias, and unfairness. Based on this corpus, we devise a recurrent neural network architecture to learn classification knowledge for bias detection. We choose this network class because of its proven ability to capture semantic information at multiple levels: taking the model output for whole texts, we conduct an in-depth reverse feature analysis to explore media bias at the word, the sentence, the paragraph, and the discourse level. At the word level, we correlate the most biased sentences with LIWC categories (Pennebaker et al., 2015). At 
the sentence and paragraph level, we reveal what parts of an article are typically most politically biased and unfair. At the discourse level, we reveal common sequential media bias patterns.

The results show that our model can utilize highlevel semantic features of bias, and it confirms that they are manifested in larger granularity levels, i.e., on the paragraph level and the discourse level. At the word level, we find some LIWC categories to be particularly correlated with political bias and unfairness, such as negative emotion, focus present, and percept. At levels of larger granularity, we observe that the last part of an article usually tends to be most biased and unfairest.

\section{Related Work}

Computational approaches to media bias focus on factuality (Baly et al., 2018), political ideology (Iyyer et al., 2014), and information quality (Rashkin et al., 2017). Bias detection has been done at different granularity levels: single sentences (Bhatia and Deepak, 2018), articles (Kulkarni et al., 2018), and media sources (Baly et al., 2019). Recently, the authors of this paper studied how the two granularity levels "sentence" and "discourse" affect each other. An outcome of this research are insights and means of how sentence-level bias classifiers can be leveraged to better predict article-level bias (Chen et al., 2020).

Given these results, the paper in hand digs deeper by investigating how bias is actually manifested in a text at different granularity levels. Our approach resembles techniques where the attention mechanism in a model is used to output weights (which indicate feature importance) for each text segment (Bahdanau et al., 2014). Zhou et al. (2016), for instance, use word-level attention to focus on sentiment words, while Ji et al. (2017) use sentencelevel attention to select valid sentences for entity relation extraction. Related to media bias, Kulkarni et al. (2018) show that the learned attention can focus on the biased sentences when detecting political ideology.

Regarding "attention" at multi-level granularity, Yang et al. (2016) propose a hierarchical attention network for document classification that is used at both word and sentence level. Yet, problems when using attention for such analyses are that the analysis unit (be it a word or a sentence) has to be defined before the training process, and that the set of classifiers which can output attention is limited. By contrast, our unsupervised reverse feature analysis can be used with any classifier and at an arbitrary semantic level, even after the training process.

\section{Media Bias Corpus}

Although bias detection is viewed as an important task, we could not find any reasonably sized labeled corpus that suits our study, which is why we decided to built a new one. We started with the corpus of Chen et al. (2018) in order to obtain news articles with topics and political bias labels. We extended the corpus by crawling the latest articles from allsides.com and by adding the fairness labels provided by adfontesmedia.com. The new corpus is available at https://github.com/ webis-de/NLPCSS-20.

Allsides.com This website is a news aggregator that collects news articles about American politics, starting from June 1st, 2012. Each event comes with articles representing one of the three political camps: left, center, and right. Chen et al. (2018) crawled the website to extract 6447 articles. For our study, we extended their corpus by integrating all articles until March 15th, 2019, resulting in a total of 7775 articles. In addition to the political bias labels, we crawled the topic tags of each article.

Adfontesmedia.com Since allsides.com focuses on political bias, we exploit adfontesmedia.com as another source for additional bias types. This portal maintains a "bias scale" quantifying the media bias of a broad set of US news portals. The bias assessments stem from media experts who annotate each portal with bias and fairness labels. Bentley et al. (2019) show that the portals' labels are highly correlated to findings from social scientists.

A New Media Bias Corpus Based on the labels from adfontesmedia.com, we define three media bias types for news portals:

1. Political Bias. A portal is neutral if it is labeled as "skew left/right" or "neutral". It is politically biased if it is labeled with "most extreme left/right" or "hyperpartisan left/right".

2. Unfairness. A portal is considered fair if it is labeled as "original fact reporting", "fact reporting", "mix of fact reporting and analysis", "analysis", or "opinion". The portal is considered unfair if it is labeled as "selective story", "propaganda", or "fabricated info". 


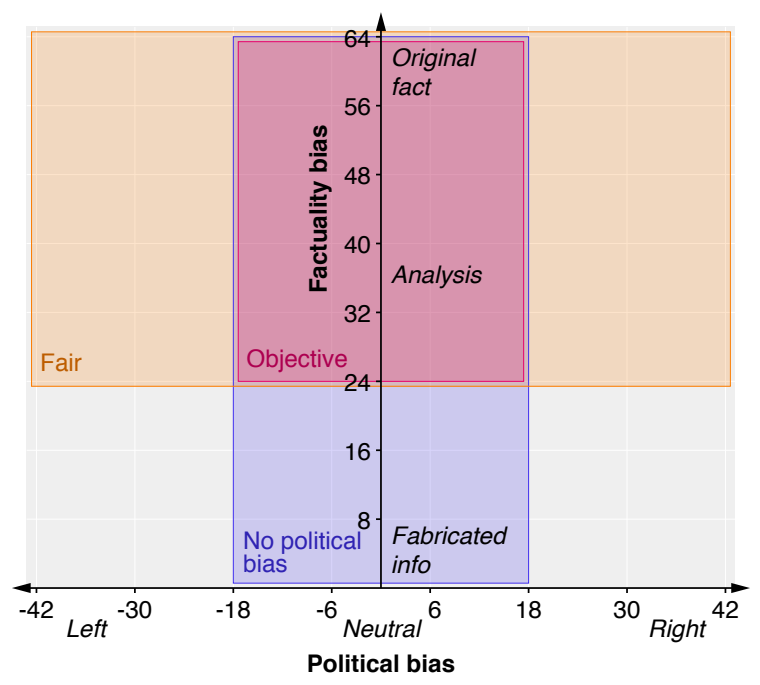

Figure 1: The bias chart from adfontesmedia.com (visually adjusted). The three rectangles represent the positive counterparts of the regions of the three bias types (political bias, unfairness, and non-objectivity).

\begin{tabular}{lrllr}
\hline \multicolumn{2}{c}{ Portal } & & & \multicolumn{2}{c}{ Topic } \\
\cline { 1 - 2 } Name & Count & & Name & Count \\
\hline CNN & 1021 & & presidential election & 914 \\
Fox News & 1002 & & politics & 525 \\
New York Times & 781 & & White House & 515 \\
$\ldots$ & & & $\ldots$ & \\
NPR News & 1 & & domestic policy & 2 \\
The Nation & 1 & EPA & 1 \\
Vice & 1 & & women's issues & 1 \\
\hline
\end{tabular}

Table 1: The top three and the bottom three portals along with the topics in our corpus.

3. Non-Objectivity. A portal is considered $o b$ jective if it is politically unbiased and fair. Otherwise, it is considered as non-objective.

Figure 1 gives an overview of the labels; it is based on the bias chart at adfontesmedia: The political bias focuses on the $\mathrm{x}$-axis of the chart, while the unfairness focuses on the $y$-axis of the chart. The non-objectivity is the combination of the two kinds of bias.

We label the collected articles according to this scheme. Since adfontesmedia.com does not cover all portals from allsides.com, the final corpus contains 41 portals with 6964 articles. The three largest portals are CNN (1021 articles), Fox News (1002 articles), and the New York Times (781 articles). Altogether, we count 111 different topics such as, "presidential election" (914 articles), "politics" (525 articles), and "white house" (515 articles). Table 1 lists the top three and the bottom three portals along

\begin{tabular}{rrrr}
\hline Media Bias & Training & Development & Test \\
\hline Political Bias & $39.25 \%$ & $40.00 \%$ & $42.82 \%$ \\
Unfairness & $18.59 \%$ & $17.48 \%$ & $18.16 \%$ \\
Non-objectivity & $39.84 \%$ & $40.66 \%$ & $43.31 \%$ \\
\hline
\end{tabular}

Table 2: The percentage of articles with each considered media bias type in the three datasets of our corpus.

with topics in our corpus.

\section{Media Bias Analysis}

This section presents our approach to study bias and unfairness at different levels of text granularity. At first, we develop classifiers for political bias, unfairness, and non-objectivity. Second, we do a reverse feature analysis study to explore the manifestation of bias at multiple levels of granularity and to identify different bias patterns.

\subsection{Media Bias Classification}

For the detection of bias in a text, we follow Chen et al. (2018) in keeping the word order, in order to be able to capture higher-level semantics. To this end, we develop classifiers with RNN served as the classical model for sequential inputs, where a cell is a GRU with a recurrent state size of 32. On top of the final hidden vector of GRUs is a prediction layer whose activation function is a softmax and the size is 2 . We use the pre-trained word embedding of GloVe (Pennington et al., 2014) with a word embedding dimension of 50, the optimizer Adam, and a learning rate of 0.001 . We train classifiers until no improvement in the development set is observed anymore; all classifiers are of the same structure and have the same hyperparameters.

To minimize the mnemonic information induced by the article topic, we split the dataset controlling the topics as an independent variable: we group the articles by their topic and select some groups to be in the test set, some to be in the development set, and the rest to be in the training set. We ensure that either the development set or the test set has at least $10 \%$ of the articles in the whole dataset, obtaining 5394 articles in the training set, 755 articles in the development set, and 815 articles in the test set.

Table 2 shows the distribution of the labels in the corpus. To avoid the exploitation of portal-specific features, each article is thoroughly checked and all information regarding the portal it was taken from (e.g., "CNN's Clare Foran and Phil Mattingly contributed to this report") is removed. 


\subsection{Reverse Feature Analysis}

In addition to the effectiveness of our bias classification, we want to assess the kind of features that are learned. Formally, we use the developed classifiers to output the predicted bias probability $p_{\text {art }}$ of the test articles, i.e., the probability of being politically biased, unfair, or non-objective. We iteratively remove text segments from the article and use the classifier to again predict the media bias probability $p_{a r t-i}$, where $i$ denotes the index of the text segment in the article. The media bias strength of a text segment $t_{i}$ is estimated as $p_{\text {art }}-p_{\text {art }-i}$. If a text segment is relevant for prediction, we expect to see a significant decrease from $p_{\text {art }}$ to $p_{\text {art }-i}$.

Based on this estimation of media bias strength, we design three experiments to analyze and interpret the classifiers' predictions at the following levels of text granularity:

Word level (LIWC correlations) Related research suggests that media bias is manifested at a larger granularity level, including the paragraph level (Chen et al., 2018) and the clause level (Iyyer et al., 2014). To validate this, we use the LIWC categories to check the word level bias, because they have been used in Iyyer et al. (2014) to sample a set of sentences that may contain ideology bias.

In detail, for each sentence $s_{i}$, we compute its LIWC score of the category $j$ as $\mid\left\{w_{i, k} \in c_{j}, k \in\right.$ $K\}|/|\left\{w_{i, k}, k \in K\right\} \mid$, where $c_{j}$ denotes the words in LIWC category $j, K$ denotes the bag-of-words in $s_{i}$, and $w_{i, k}$ denotes the $k$-th word in $K$. The Pearson correlation coefficient is used to measure the correlation between LIWC categories and media bias strength.

Sentence and paragraph-level (locations of media bias) Here we analyze the distribution of the media bias strength in the sentences and paragraphs (approximated as three continuous sentences). Basically, these values indicate which segment of a text mostly contains media bias.

Discourse level (media bias patterns) Here we analyze the patterns of the media bias strength across the different parts of an article's discourse. In particular, we split an article into four equallysized parts and compute the average media bias strength of the sentences for each part. The splitting is comparable to the so-called "inverted pyramid" structure in journalism, where a news article starting by the summary, important details, general and background info (Pöttker, 2003).

\begin{tabular}{lrrr}
\hline & Political Bias & Unfairness & Non-objectivity \\
\hline Majority & $36.38 \%$ & $45.01 \%$ & $36.18 \%$ \\
RNN & $75.60 \%$ & $83.42 \%$ & $75.42 \%$ \\
- Biased & $69.41 \%$ & $72.09 \%$ & $69.57 \%$ \\
- Unbiased & $81.80 \%$ & $94.75 \%$ & $81.13 \%$ \\
\hline
\end{tabular}

Table 3: The $\mathrm{F}_{1}$ scores of RNN, majority baseline, and by-class performance of the three bias types.

\section{Results and Discussion}

In the following, we report and discuss the results of the experiments described in Section 4.

\subsection{Media Bias Classification}

First, we look at the automatic classification of media bias. Table 3 summarizes the performance of the developed RNNs in the three media bias classes. All classifiers outperform the majority baseline, achieving $75.60 \%$ for political bias, $83.42 \%$ for unfairness, and $75.42 \%$ for non-objectivity. Such a performance demonstrates the capability of the classifiers to detect topic-independent media bias features.

Looking closely at individual bias classes, we find that the RNN is good at predicting the absence of bias rather than bias. We interpret this because of the uneven distribution of the classes, especially in the unfairness (see Table 2).

\subsection{Reverse Feature Analysis}

Although the classifiers achieved good results, they are certainly not perfect. To account for the prediction errors, the following analysis uses only the predicted probabilities of those articles where the RNNs predicted the label correctly.

LIWC Correlations According to the Pearson correlations, most of the LIWC categories are not correlated with a high coefficient (neither in a positive or a negative way). However, the highest correlated categories are different among the three types of media bias. The categories that have the highest correlation with political bias are negative emotion, anger, and affect. This shows that politically biased articles tend to use emotional and opinionated words such as "disappoint", "trust", and "angry". For unfair articles, we see a higher correlation in focus present. Examples in this category are "admit", "become", and "determine". For non-objective articles, the bias is related to percept words such as "feel", "gloom", and "depict". 


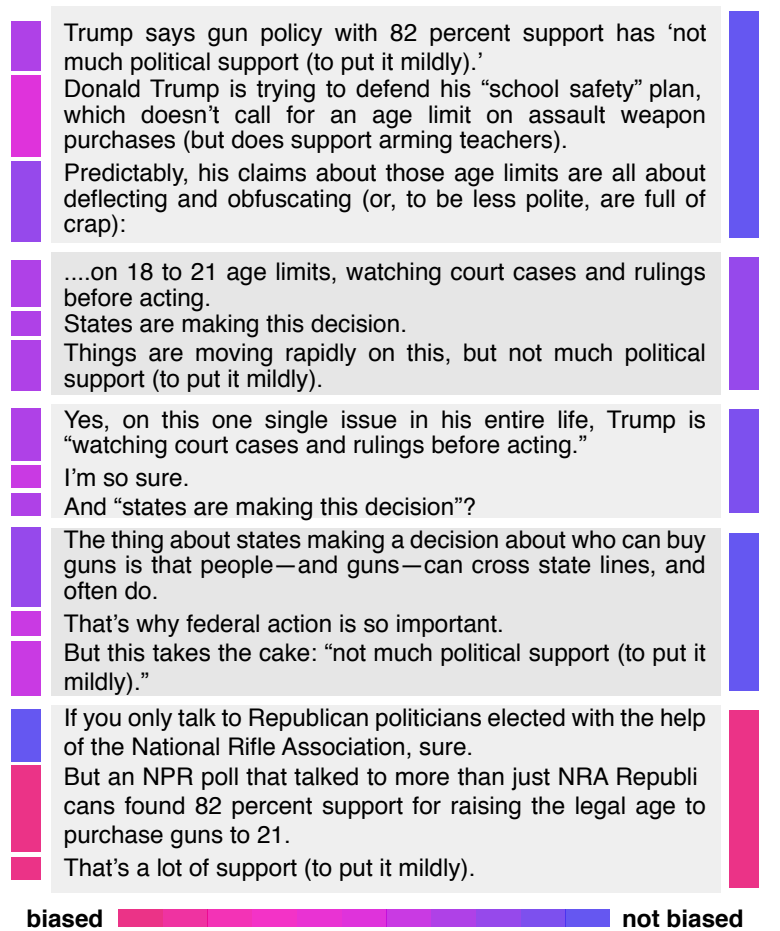

Figure 2: The media bias strength on sentence (left) and paragraph (right) level in an excerpt of one news article from the given corpus. Biased text segments are shown in red and unbiased text segments in blue.

Location of Media Bias Figure 2 visualizes the estimated media bias strength at the sentence and paragraph levels. As an example, we choose an article from Daily Kos, which is labeled as politically biased. In this article, we see a strong tendency to criticize Trump's claim, especially at the end of the article. At the paragraph level, our strength analysis of media bias successfully identifies the last paragraph as the most biased text segment. While at the sentence level, we see that the last two sentences are most biased in the last paragraph. The second sentence seems to be a bit biased, perhaps because of the word usage of "trying to defend". However, we see that the analysis fails to identify the third sentence as politically biased. Still, given that the sentence or paragraph level analysis is fully unsupervised, the reverse feature analysis seems to perform quite well.

Media Bias Patterns Figure 3 shows the identified media sequential patterns for the three bias types. We can notice that the media bias strengths for all articles in the second quarter are somewhat close. This is, in our opinion, because the second quarter of news articles usually contains some background information, which does not tend to

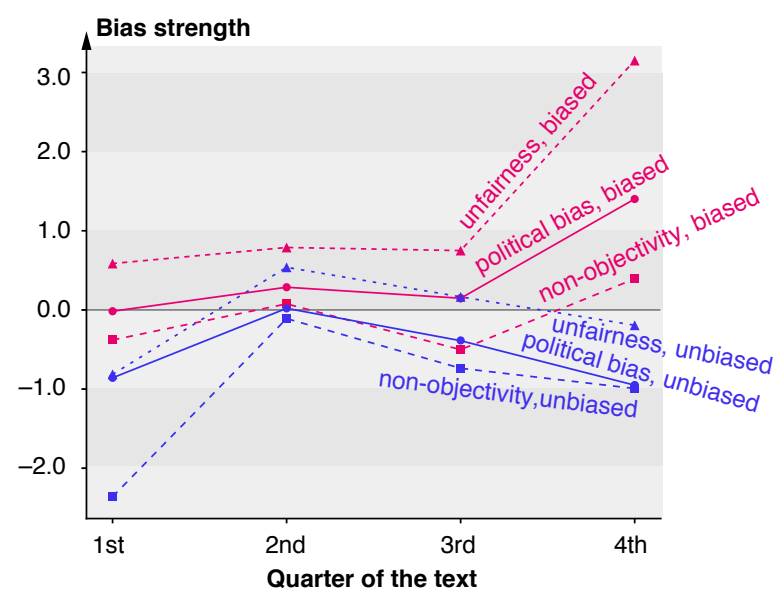

Figure 3: Patterns of the types of media bias as well as biased and unbiased text. Values are normalized to have a mean of zero and a standard deviation of one. Positive values indicate a stronger bias and negative values indicate that text has a lower bias or is unbiased.

be biased. We also see that all biased articles start with a neutral tone (close to mean) in the beginning and then emphasize the bias in the latter parts. Among the three media bias types, unfairness has the highest bias strength. Observing our corpus, we find that one typical way to be unfair is to report selected facts in a favor of some entity, which leads to completely different word usage. On the other hand, for political bias, describing facts with positive or negative expressions is a common indicator of bias there. Such a difference might be the reason for why the classifiers can better discover the unfair texts.

\section{Conclusion}

This paper has studied political bias and unfairness in news articles. We have trained sequential models for bias detection and have applied a reverse feature analysis to demonstrate that it is possible to reveal at what granularity level and how sequential patterns media bias is manifested. Specifically, we find that the last quarter seems to be the most biased part. A significant "by-product" of our research is a new corpus for bias analysis. And we believe this corpus can help, for example, investigate how journalists convey bias in a news article.

In the future, we will study how to utilize these results to detect bias or to rewrite the articles to remove and change bias. For example, based on the findings of this paper, the bias remover should pay more attention to the later paragraphs. 


\section{Acknowledgments}

This work was partially supported by the German Research Foundation (DFG) within the Collaborative Research Center "On-The-Fly Computing" (SFB 901/3) under the project number 160364472.

\section{References}

Dzmitry Bahdanau, Kyunghyun Cho, and Yoshua Bengio. 2014. Neural machine translation by jointly learning to align and translate. arXiv preprint arXiv:1409.0473.

Ramy Baly, Georgi Karadzhov, Dimitar Alexandrov, James Glass, and Preslav Nakov. 2018. Predicting factuality of reporting and bias of news media sources. In Proceedings of the 2018 Conference on Empirical Methods in Natural Language Processing, pages 3528-3539.

Ramy Baly, Georgi Karadzhov, Abdelrhman Saleh, James Glass, and Preslav Nakov. 2019. Multi-task ordinal regression for jointly predicting the trustworthiness and the leading political ideology of news media. arXiv preprint arXiv:1904.00542.

Frank Bentley, Katie Quehl, Jordan Wirfs-Brock, and Melissa Bica. 2019. Understanding online news behaviors. In Proceedings of the 37th Annual ACM Conference on Human Factors in Computing Systems.

Sumit Bhatia and P Deepak. 2018. Topic-specific sentiment analysis can help identify political ideology. In Proceedings of the 9th Workshop on Computational Approaches to Subjectivity, Sentiment and Social Media Analysis, pages 79-84.

Wei-Fan Chen, Khalid Al Khatib, Benno Stein, and Henning Wachsmuth. 2020. Detecting media bias in news articles using gaussian bias distributions. In Proceedings of the 2020 Conference on Empirical Methods in Natural Language Processing, Findings of ACL: EMNLP 2020. To appear.

Wei-Fan Chen, Henning Wachsmuth, Khalid Al Khatib, and Benno Stein. 2018. Learning to flip the bias of news headlines. In Proceedings of the 11th International Conference on Natural Language Generation, pages 79-88.

Tim Groseclose and Jeffrey Milyo. 2005. A measure of media bias. The Quarterly Journal of Economics, 120(4):1191-1237.

Mohit Iyyer, Peter Enns, Jordan Boyd-Graber, and Philip Resnik. 2014. Political ideology detection using recursive neural networks. In Proceedings of the 52nd Annual Meeting of the Association for Computational Linguistics, volume 1, pages 1113-1122.
Guoliang Ji, Kang Liu, Shizhu He, and Jun Zhao. 2017. Distant supervision for relation extraction with sentence-level attention and entity descriptions. In Proceedings of the Thirty-First AAAI Conference on Artificial Intelligence.

Vivek Kulkarni, Junting Ye, Steve Skiena, and William Yang Wang. 2018. Multi-view models for political ideology detection of news articles. In Proceedings of the 2018 Conference on Empirical Methods in Natural Language Processing, pages 3518 3527.

James W Pennebaker, Ryan L Boyd, Kayla Jordan, and Kate Blackburn. 2015. The development and psychometric properties of LIWC2015.

Jeffrey Pennington, Richard Socher, and Christopher Manning. 2014. GloVe: Global vectors for word representation. In Proceedings of the 2014 Conference on Empirical Methods in Natural Language Processing, pages 1532-1543.

Horst Pöttker. 2003. News and its communicative quality: The inverted pyramid — when and why did it appear? Journalism Studies, 4(4):501-511.

Hannah Rashkin, Eunsol Choi, Jin Yea Jang, Svitlana Volkova, and Yejin Choi. 2017. Truth of varying shades: Analyzing language in fake news and political fact-checking. In Proceedings of the 2017 Conference on Empirical Methods in Natural Language Processing, pages 2931-2937.

Zichao Yang, Diyi Yang, Chris Dyer, Xiaodong He, Alex Smola, and Eduard Hovy. 2016. Hierarchical attention networks for document classification. In Proceedings of the 2016 Conference of the North American Chapter of the Association for Computational Linguistics: Human Language Technologies, pages 1480-1489.

Xinjie Zhou, Xiaojun Wan, and Jianguo Xiao. 2016. Attention-based lstm network for cross-lingual sentiment classification. In Proceedings of the 2016 Conference on Empirical Methods in Natural Language Processing, pages 247-256. 